\title{
ESTUDIOS FENOMORFOLÓGICOS EN LA VEGETACIÓN DEL SUR DE ESPAÑA. II. ALCORNOCALES MESOMEDITERRÁNEOS. (MONTES DE MÁLAGA, MÁLAGA)
}

\author{
Baltasar CABEZUDO, Andrés V. PÉREZ LATORRE, \\ Teresa NAVARRO y José M. NIETO CALDERA
}

\begin{abstract}
RESUMEN. Estudios fenomorfológicos en la vegetación del sur de España. II. Alcornocales mesomediterráneos. (Montes de Málaga, Málaga). Se describe el comportamiento fenomorfológico de una formación vegetal incluíble en la serie de los alcornocales mesomediterráneos con encinas del sector Malacitano-Axarquiense (Málaga, España). Se realizó un análisis basado en el estudio de las distintas fenofases para los táxones persistentes de la comunidad. Se tomaron para ello los datos de inicio y mantenimiento de cada fenofase (prefloración, floración, fructificación, dispersión, caída de hojas, crecimiento vegetativo) para cada especie y algunos caracteres ecomorfológicos como tipo biológico, estacionalidad, tipo de fruto y tipo de hoja. Como resultado se han caracterizado las estaciones más favorables o desfavorables para la comunidad y la relación entre las fenofases y el clima local. Como conclusión, la estación más favorable para la vegetación estudiada es la Primavera, siendo la más desfavorable el Verano, comportándose la comunidad como multiestacional. Se ha detectado un contingente de especies que muestran un comportamiento distinto respecto a la norma general de la comunidad.
\end{abstract}

Palabras clave. Fenomorfología, fenofases, alcornocales, Andalucía, España.

ABSTRACT. Phenomorphological studies in south of Spain vegetation. II. Meso-mediterranean Quercus suber woods. (Montes de Málaga, Málaga). A phenomorphological analysis based on the study of phenological phases for persisting species in a Q. suber community was carried out. In order to do this, data from starting and maintenance of phenophases were taken for each species (flower buds formation, flowering, fruit setting, seed dispersal, brachyblast vegetative growth, dolichoblast vegetative growth, leaf shedding brachyblast and leaf shedding dolichoblast) as well as some ecomorphological characters: life form, seasonality, fruit type and leaf consistency. As an outcome, more favourable and unfavourable seasons for the community and the relation among phenological phases and local climate were characterized. Spring is the most favourable season for the community while Summer is the most unfavourable one. Vegetation shows multiseasonal phenological behaviour. Some species show different phenological pattern from community general pattern.

Key words. Phenomorphology, phenological phases, Quercus suber woods, Andalusia, Spain.

\section{INTRODUCCIÓN}

La fenomorfología (descripción de los cambios estacionales en la morfología de las plantas durante su ciclo completo de vida) intenta relacionar, mediante el estudio de órganos de la planta (hojas, tipos de yemas, flores, braquiblastos, dolicoblastos, etc.) y fenofases (prefloración, floración, fructificación, dispersión, caída de hojas, etc.), 
el comportamiento de plantas o de comunidades y su adaptación al medio donde se desarrollan (Orshan, 1982 y 1989a). El patrón de distribución de los caracteres fenomorfológicos principales está relacionado con el funcionamiento de las plantas y sus adaptaciones al medio (Le Roux et al, 1984; Pierce, 1984, Mooney et al, 1983); este patrón es un atributo (Floret et al, 1990) comparable al espectro de tipos biológicos que se puede deducir de un clima local (Halloy, 1983, 1990). El uso de la fenomorfología para el estudio de las comunidades vegetales puede ser complementario al fitosociológico de Braun-Blanquet (1964) basado en la composición florística (Floret et al op. cit.).

Este método puede ser aplicado a áreas pequeñas y ser efectivo en muchos casos para dilucidar procesos funcionales de los ecosistemas (Floret et al op. cit.). Existe muy poca información relativa a estudios fenomorfológicos a nivel mundial (Orshan, 1989a) y a nivel de Andalucía (Cabezudo et al, 1992). En este trabajo se intenta caracterizar una formación vegetal mediante su comportamiento fenomorfológico (fenofases); para ello se eligió un alcornocal en la provincia de Málaga (Venta Lanada, Montes de Málaga, coord. U.T.M. 30SUF8078). Esta zona se en-

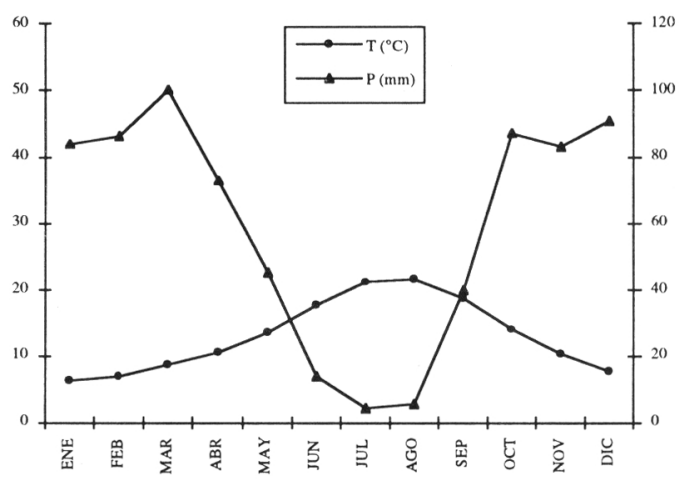

Figura 1. Diagrama ombrotérmico. Hydrothermic diagram. Lagar de Torrijos, 780 m.s.m.. Montes de Málaga. Málaga. cuentra entre 800 y 900 m. s. m., y presenta fuertes pendientes y orientación norte; litológicamente dominan las pizarras, cuarcitas y grauwakas; los suelos corresponden, donde el bosque se halla conservado, a luvisoles existiendo también cambisoles eútricos y litosoles. Según los datos de la estación climática mas cercana (fig. 1), la zona se incluye dentro del piso bioclimático mesomediterráneo con ombroclima subhúmedo, la temperatura media anual es de $13^{\circ}$, la precipitación media anual está entre 700 y $800 \mathrm{~mm}$. lo que condiciona un Ia dentro de la región higrófita. Respecto al índice de continentalidad (K) la zona se incluye dentro de áreas algo continentales, el período seco oscila entre 3 y 3,5 meses, el período frío es de 4 meses y el tipo fitoclimático es $\mathrm{IV}_{4}$. Se contabilizan 2.921 horas de sol anuales (fig. 2).

La vegetación potencial del territorio corresponde a los alcornocales con encinas de distribución Malacitano-Axarquiense de la serie Teucrio-Querceto suberis quercetoso rotundifoliae S (Nieto et al, 1991). El uso del territorio, basado en cultivos marginales de secano, ha traído como consecuencia la aparición de jarales (Lavandulo-Genistetum equisetiformis) y pastizales nitrófilos (InuloOryzopsietum miliaceae) siendo más escasas

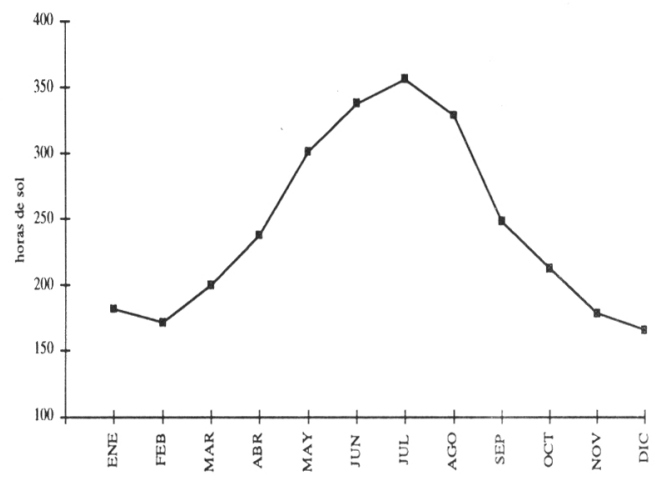

Figura 2. Horas mensuales de sol en Málaga. Monthly insolation in Málaga. 
las formaciones boscosas de alcornocal (Teucrio-Quercetum suberis quercetosum rotundifoliae) y sus madroñales (CytisoArbutetum unedi bupleuretosum fruticosi) (Pérez Latorre et al, 1993).

\section{METODOLOGÍA}

El análisis fenológico se ha realizado según la metodología de Orshan (1989b), para lo cual se seleccionó una parcela representativa de la vegetación de la zona donde se realizó un inventario de las especies presentes dividiéndolas, segun Evenari et al (1975), en persistentes (árido-activas) y efímeros y efemeroides (árido-pasivas), en función de la persistencia o no de la parte aérea. El seguimiento se llevó a cabo sólo sobre las 36 especies persistentes, para lo cual y durante un periodo de 18 meses (1990-1992) se visitó la parcela cada 3-4 semanas anotando los datos cualitativos de inicio y mantenimiento de cada fenofase reproductiva y vegetativa para cada especie. En cada muestreo se recolectaron pliegos significativos del periodo fenofásico en que se encontraba cada especie lo que permitió su estudio en laboratorio. Como datos complementarios se estudiaron algunos caracteres ecomorfológicos (Orshan, 1986): estacionalidad, tipo de fruto, tipo de hoja y tipo biológico.

\section{RESULTADOS}

Los resultados obtenidos se reflejan en la tabla 1 donde para cada especie se indican los periodos de inicio y finalización de las distintas fases fenológicas reproductivas: formación de yemas florales (PF), floración (FL), fructificación (FR) y dispersión (DS) y vegetativas: crecimiento vegetativo del braquiblasto (CVB), crecimiento vegetativo del dolicoblasto (CVD), caída de hojas del braquiblasto (CHB) y caída de hojas del dolicoblasto (CHD). Se indican además algunos caracteres directamente relacionados con la duración de algunas de estas fenofases tales como estacionalidad (EST), tipo de fruto, tipo de hoja y tipo biológico.

A partir de los datos de la tabla 1 se han realizado una serie de gráficas que nos muestran el comportamiento general de la comunidad (en porcentaje) respecto al inicio de las fenofases (figs. 3 y 4 ) y al porcentaje de especies que presentan las distintas fenofases en cada mes (figs. 5 y 6). En base a dichos resultados se comentan los aspectos mas significativos de cada fenofase.

La estación en la que inician la prefloración (PF) el mayor número de especies (fig. 3) es la Primavera con un total de 21, destacando el mes de Abril con 9; le siguen en importancia el Invierno con 8, el Verano con 5 y el Otoño con sólo 2 especies (Viburnum tinus y Ulex parviflorus). La estación en la que se acumulan mayor número de especies en prefloración es la Primavera (fig. 5), destacando los meses de Abril y Mayo con un total de 21 especies; por el contrario, la estación con menor número de especies en prefloración es el Otoño donde destaca Noviembre con sólo 2 (Viburnum tinus y Ulex parviflorus). El período entre el inicio de la prefloración y la floración varía entre las especies estudiadas, siendo el más característico el de un mes entre ambas fenofases (18 especies), existiendo 6 con 2 meses de intervalo, 4 con 3 meses, 1 con 4 meses (Arbutus unedo), 1 con 6 meses (Viburnum tinus) y 6 especies que florecen en el mismo mes en que inician la prefloración. Otra característica diferencial de las especies estudiadas es el período total en que cada una de ellas se encuentra en prefloración, siendo el de 2 a 3 meses el más característico de la comunidad estudiada, destacando los 8 meses de prefloración de Viburnum tinus (tab. 1).

La floración (FL) se inicia preferentemente en Primavera (fig. 3) con un total de 23 


\begin{tabular}{|c|c|c|c|c|c|c|c|c|c|c|c|c|}
\hline \multirow[t]{2}{*}{ ESPECIES } & \multicolumn{8}{|c|}{ FENOFASES } & \multicolumn{4}{|c|}{ C. ECOMORFOLÓGICOS } \\
\hline & $\mathbf{P F}$ & FL & FR & DS & CVB & CVD & $\mathrm{CHB}$ & CHID & EST & TF & TH & $\mathrm{TB}$ \\
\hline Centaurea sempervirens & $\mathrm{V}-\mathrm{VI}$ & VI-VIII & VII-IX & VIII-X & - & $\mathrm{I}-\mathrm{V}$ & - & VII-XII & $S$ & Aquenio & $M$ & $\mathrm{Ca}$ \\
\hline Cistus salvifolius & II-IV & IV-VI & VI-IX & IX-XI & IX-VI & V-IX & V-VII & VI-X & $S$ & Cápsula & M & $\mathrm{Ca}$ \\
\hline Genista umbellata & IV-VI & VI-VII & VI-VII & VII-VIII & - & III-V & - & & $S$ & Legumbre & & $\mathrm{Ca}$ \\
\hline Inula viscosa & V-VIII & VI-IX & VII-X & VIII-XI & XII-III & IV-IX & - & VII-IX & S & Aquenio & M & $\mathrm{Ca}$ \\
\hline Lavandula stoechas & II-IV & IV-VI & VI-VIII & IX-XII & I-XII & IX-VI & I-XII & VII-VIII & $\mathrm{S}$ & Núcula & $\mathrm{M}$ & $\mathrm{Ca}$ \\
\hline Origanum virens & VI & VI-VII & VII & VIII-IX & $X-V$ & V-VI & - & VI-IX & $\mathrm{S}$ & Núcula & M & $\mathrm{Ca}$ \\
\hline Phlomis purpurea & III-V & IV-VI & V-VII & VI-VIII & I-XII & IX-IV & I-XII & VII-VIII & $\mathrm{S}$ & Cápsula & M & $\mathrm{Ca}$ \\
\hline Ptilostemon hispanicus & V-VI & VI-VII & VII-IX & VII-IX & $\mathrm{XI}-\mathrm{V}$ & III-VIII & $\mathrm{XI}-\mathrm{V}$ & $\mathrm{I}-\mathrm{XII}$ & S & Aquenio & $\mathrm{S}$ & $\mathrm{Ca}$ \\
\hline Rubia peregrina & IV-VI & IV-VI & VI-XI & IX-XI & - & III-VI & - & - & $\mathrm{s}$ & Drupa & $\mathrm{s}$ & $\mathrm{Ca}$ \\
\hline Sanguisorba hybrida & IV-V & v & V & VI-VII & XII-IV & IV-V & V-VII & VIII-IX & $S$ & Núcula & M & $\mathrm{Ca}$ \\
\hline Doronicum plantagineum & III-IV & IV-V & V-VI & V-VI & - & I-IV & - & $\mathrm{V}-\mathrm{VI}$ & D & Aquenio & M & $\mathrm{Cr}$ \\
\hline Quercus faginea & V & V & V-X & $\mathrm{X}-\mathrm{XII}$ & - & IV-VI & - & XII-II & $\mathrm{D}$ & Glande & $\mathrm{S}$ & meF \\
\hline Quercus rotundifolia & IV-V & $\mathrm{V}-\mathrm{VI}$ & $\mathrm{V}-\mathrm{X}$ & $\mathrm{XI}-\mathrm{I}$ & - & IV-VI & - & - & S & Glande & $\mathrm{E}$ & \\
\hline Quercus suber & III-VI & IV-VI & VI-XI & $X-I$ & - & $\mathrm{V}$ & - & V-VI & $\mathrm{S}$ & Glande & $\mathrm{S}$ & meF \\
\hline Arbutus unedo & VII-X & XI-I & I-XII & X-XII & - & III-VII & - & VI-VII & S & Baya & S & $\mathrm{mF}$ \\
\hline Crataegus monogyna & IV-V & $\mathrm{V}$ & VI-IX & IX-XII & II-IV & V & - & IX-XI & D & Pomo & M & $\mathrm{mF}$ \\
\hline Erica arborea & $\mathrm{I}-\mathrm{V}$ & IV-VI & V-VII & VII-IX & I-XII & VI-XI & VII-VII & IVIII-VIII & $\mathrm{S}$ & Cápsula & E & $\mathrm{mF}$ \\
\hline Juniperus oxycedrus & I-V & III-V & 2 años & II-V & - & III-VI & - & VI & $\mathrm{S}$ & Gálbulo & E & $\mathrm{mF}$ \\
\hline Phyllirea angustifolia & XII-III & III-IV & IV-IX & IX-X & - & V-VI & - & IV-V & S & Drupa & S & $\mathrm{mF}$ \\
\hline Quercus coccifera & V-VI & VI-VII & 1.5 años & IX-XI & - & V/X-XII & - & VI-VII & $S$ & Glande & E & $\mathrm{mF}$ \\
\hline Viburnum tinus & IX-IV & III-IV & V-X & $\mathrm{X}-\mathrm{XII}$ & - & IV-VII & - & VI-VII & S & Drupa & $S$ & $\mathrm{mF}$ \\
\hline Adenocarpus telonensis & V-VII & VI & VI-VIII & VII-IX & III-VI & XII-V & & V-IX & S & Legumbre & M & $\mathrm{nF}$ \\
\hline Bupleurum fruticosum & VI-VII & VIII-VIII & VIII-IX & VIII-X & - & II-V & - & XII-IV & S & Mericarpo & $\mathrm{S}$ & $n F$ \\
\hline Cistus albidus & III-V & IV-V & V-VIII & IX-XII & I-XII & XII-IV & I-XII & VI-VIII & $\mathrm{S}$ & Cápsula & $\mathrm{M}$ & $\mathrm{nF}$ \\
\hline Cistus ladanifer & I-IV & IV-V & VI-VIII & VIII-XII & I-XII & V-IX & I-XII & VI-VIII & $\mathrm{S}$ & Cápsula & M & $\mathrm{nF}$ \\
\hline Cistus monspelliensis & IV-V & IV-V & V-VIII & VIII-XI & $X-I V$ & IV-XI & VI-VII & IV-VIII & S & Cápsula & $M$ & $n F$ \\
\hline Cistus populifolius major & IV-V & V-VI & VI-VIII & VIII-XI & XII-III & III-VIII & II-IV & VI-VIII & S & Cápsula & M & $n F$ \\
\hline Cytisus grandiflorus & III-IV & $\mathrm{V}$ & V-VII & VII-IX & II-III & III-VII & - & VI-VIII & S & Legumbre & M & $\mathrm{nF}$ \\
\hline Lonicera implexa & V-VI & VI-VII & VII-IX & IX-X & - & III-V & - & VI-XII & $\mathrm{S}$ & Baya & $\mathrm{S}$ & $n F$ \\
\hline Retama sphaerocarpa & XII-III & III-IV & IV-IX & IX-X & - & V-VI & - & - & S & Legumbre & - & $n F$ \\
\hline Rosa canina & IV $-\mathrm{V}$ & IV-V & V-IX & X-IV & - & III-VII/IX-XI & - & X-II & $\mathrm{D}$ & Cinorrodon & M & $n F$ \\
\hline Teucrium fruticans & II-IV & III-VI & V-VII & VI-VIII & I-XII & VIII-XI/III-V & I-XII & VII-VIII & $S$ & Núcula & M & $\mathrm{nF}$ \\
\hline Ulex parviflorus & $\mathrm{X}$-III & XI-V & VI-VIII & VII-IX & - & V-IX & - & - & S & Legumbre & & $\mathrm{nF}$ \\
\hline Scrophularia scorodonia & IV-V & $\mathrm{V}$ & VI-VIII & VI-IX & XII-II & II-V & - & VI-X & S & Cápsula & M & \\
\hline Rubus ulmifolius & VII & VII-VIII & VIII-IX & IX-X & - & $\mathrm{V}-\mathrm{VI}$ & - & X-XII & $S$ & Polidrupa & M & $\mathrm{nF} / \mathrm{C}$ \\
\hline Daphne gnidium & VI-X & VIII-XI & IX-XII & XI-I & - & IV-VII & - & VII-XI & $\mathrm{S}$ & Drupa & M & $\mathrm{nF} / \mathrm{H}$ \\
\hline
\end{tabular}

Tabla 1. Fenofases y caracteres ecomorfológicos del alcornocal de los Montes de Málaga (Málaga). Fenofases reproductivas: formación de yemas florales (PF), floración (FL), fructificación (FR), dispersión (DS). Fenofases vegetativas: crecimiento vegetativo del braquiblasto (CVB), crecimiento vegetativo del dolicoblasto (CVD), caída de hojas del braquiblasto (CHB) y caída de hojas del dolicoblasto (CHD). Estacionalidad (EST): siempreverde (S), decidua (D). Tipo de hoja (TH): malacófila (M), semiesclerófila (S) y esclerófila (E). Tipobiológico (TB): caméfito (Ca), mesofanerófito (meF), microfanerófito (mF), nanofanerófito $(\mathrm{nF})$, criptófito $(\mathrm{Cr})$, hemicriptófito $(\mathrm{H})$. TF: Tipo de frutos.

Phenological phases and ecomorphological characters in the study area (Quercus suber woods). Reproductive phenophases: flower buds formation $(P F)$, flowering $(F L)$, fruit setting $(F R)$, seed dispersal $(D S)$. Vegetative phenophases: brachyblast vegetative growth $(C V B)$, dolichoblast vegetative growth (CVD), leaf shedding brachyblast (CHB) and leaf shedding dolichoblast (CHD). Seasonality $(S T)$ : evergreen $(S)$, deciduous $(D)$. Leaf consistency $(T H)$ : malacophyll $(M)$, semi-sclerophyll $(S)$ and sclerophyll $(E)$. Life form $(T B)$ : chamaephyte $(C a)$, meso-phanerophyte (me $F)$, micro-phanerophyte (mF), nano-phanerophyte $(n F)$, cryptophyte $(\mathrm{Cr})$, hemicryptophyte $(H)$. TF: fruit type. 
especies, destacando el mes de Abril con 11; en el Verano inician la floración 11 especies, destacando Junio con 8; el Otoño, con 2 especies (Arbutus unedo y Ulex parviflorus), y el Invierno, con ninguna, son los periodos menos favorables para el inicio de la floración. La estación con mayor número de especies en flor es la Primavera (fig. 5), destacando Mayo con 20; le sigue el Verano donde destaca el mes de Junio con 16 especies; la estación con menor número de especies en flor es el Invierno destacando Febrero con una sola (Ulex parviflorus). El periodo total de floración de cada especie varía entre 1 mes (6 especies) y 7 meses (Ulex parviflorus), siendo el periodo más común de 2 a 3 meses.

El inicio de la fructificación (FR) se produce fundamentalmente en el Verano (fig. 3) con un total de 19 especies, destacando Junio con 11; otro período importante para esta fenofase es el final de la Primavera con un total de 15 especies; por el contrario, Otoño e Invierno, solamente presentan una especie iniciando la fructificación. La estación en que se encuentran mayor número de especies fructificando es el Verano (fig. 5) con un máximo de 31 en Julio; el periodo menos favorable es el Invierno con 3 especies (Arbutus unedo, Quercus coccifera y Juniperus oxycedrus). El periodo entre el inicio de la fructificación y el de inicio de la dispersión en las especies varía entre más de 15 meses (Juniperus oxycedrus) y 1 mes (10 especies), siendo el período mas común el de 1 a 3 meses; con 11 meses destaca Arbutus unedo y con 15 Quercus coccifera. La duración de esta fenofase oscila entre las especies que están todo el año fructificando (Arbutus ungdo, Quercus coccifera y Juniperus oxycedrus) y las que fructifican en 1 mes (Origanum virens y Sanguisorba hybrida), siendo el periodo más común el de 3 meses (13 espécies) y el de 6 meses (7 especies).

Las estaciones con mayor número de especies que inician la dispersión (DS) son Verano y Otoño (fig. 3) con 17 especies respecti- vamente, destacando Septiembre con 10 y Agosto con 7; por el contrario, en Invierno y Primavera, sólo inician la dispersión dos especies, Doronicum plantagineum en Mayo y Juniperus oxycedrus en Febrero. La estación con mayor número de especies dispersando es el Otoño (fig. 5) destacando Septiembre con 23; el periodo con menor número de especies dispersando es la Primavera. La duración de la dispersión por especies varía entre 3 meses ( 17 especies) y 7 meses (1 especie); el periodo más común de duración de la dispersión es el de 2 a 4 meses.

El crecimiento vegetativo del dolicoblasto (CVD) se inicia preferentemente (fig. 4) en Primavera con 26 especies, destacando Mayo con 10; el periodo mas desfavorable es el Verano con 2 especies; las otras estaciones presentan valores intermedios entre 3 en Otoño y 7 en Invierno. La estación en que se presentan mayor número de especies con crecimiento del dolicoblasto (fig. 6) es la Primavera, destacando Mayo con 32 y Abril con 25; el principio del Verano es también un periodo muy activo en el que destaca Junio con 22 especies; en Otoño e Invierno decae considerablemente esta fenofase con sólo 5 especies en crecimiento durante los meses de Noviembre y Diciembre. La duración de esta fenofase oscila entre 1 mes (Quercus subery Crataegus monogyna) y 10 meses (Lavandula stoechas), siendo la más frecuente entre 4 y 6 meses.

La principal época de crecimiento vegetativo del braquiblasto (CVB) es desde Diciembre a Abril (fig. 6), mientras que el mínimo ocurre durante el Verano. El periodo de crecimiento oscila entre 2 meses (Adenocarpus telonensis) y 12 meses (Lavandula stoechas, Phlomis purpurea, Erica arborea, Cistus albidus, Cistus ladanifer y Teucrium fruticans), siendo el más común el de 12 meses.

El inicio de la caída de hojas del dolicoblasto (CHD) se produce principalmente (fig. 4) durante el Verano (20 especies), 

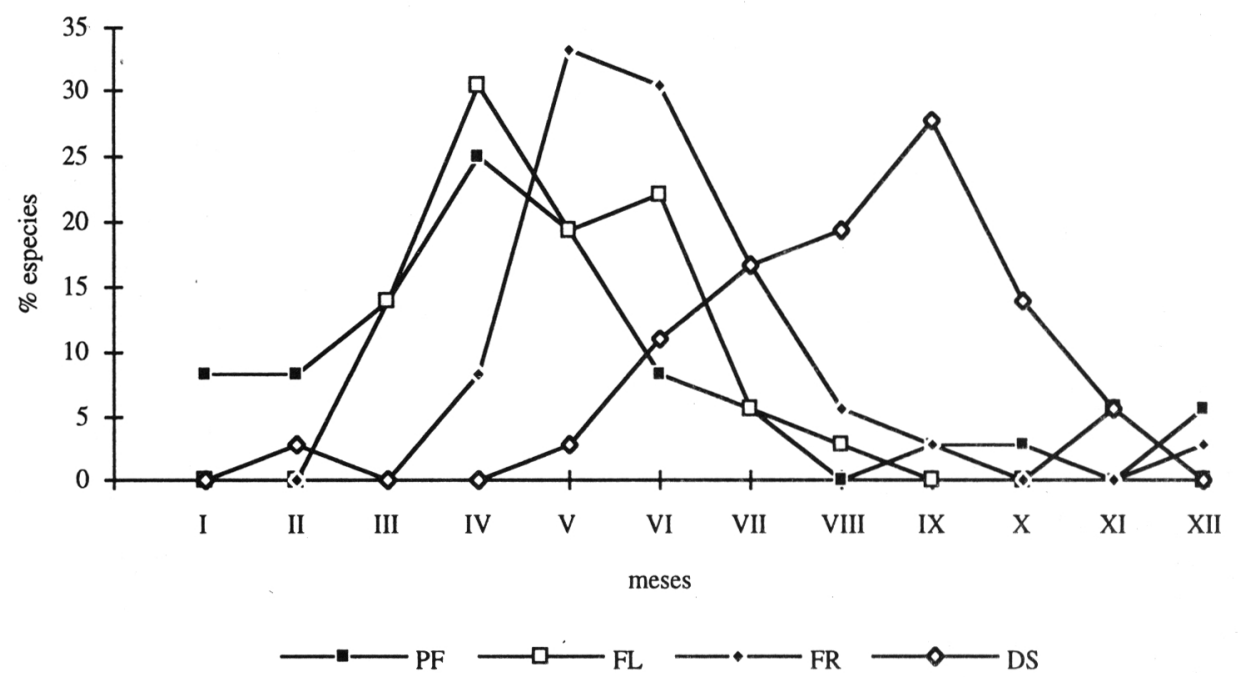

Figura 3. Porcentaje mensual de especies (\%) respecto al inicio de las fenofases reproductivas. Prefloración (PF), floración (FL), fructificación (FR) y dispersión (DS). Monthly percentage of species starting reproductive phenophases. Flower buds formation $(P F)$, flowering $(F L)$, fruit setting $(F R)$ and seed dispersal (DS).
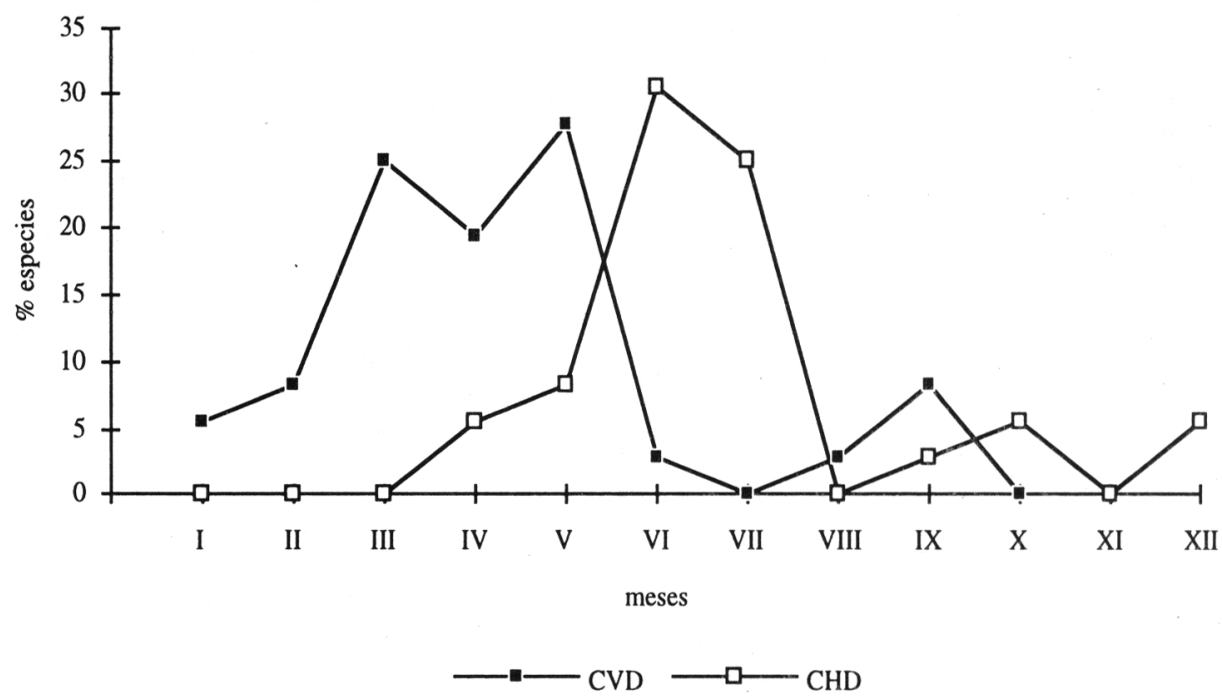

Figura 4. Porcentaje mensual de especies respecto al inicio de las fenofases vegetativas (relativas a dolicoblastos). Crecimiento vegetativo del dolicoblasto (CVD) y caída de hojas del dolicoblasto (CHD). Monthly percentage of species starting vegetative phenophases. (only dolichoblasts). Dolichoblast vegetative growth (CVD) and leaf shedding dolichoblast (CHD). 

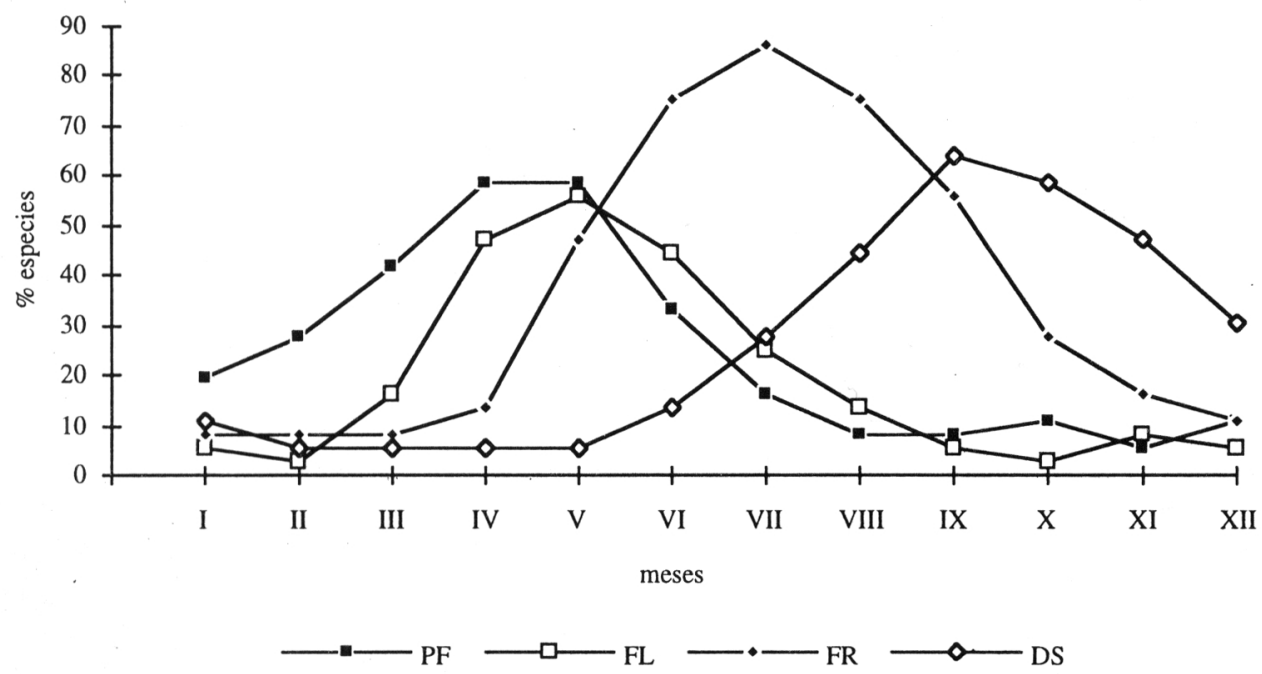

Figura 5. Porcentaje mensual de especies que presentan las fenofases reproductivas. Prefloración (PF), floración (FL), fructificación (FR) y dispersión(DS). Monthlypercentage of species showing reproductive phenophases. Flower buds formation $(P F)$, flowering $(F L)$, fruit setting $(F R)$ and seed dispersal (DS).
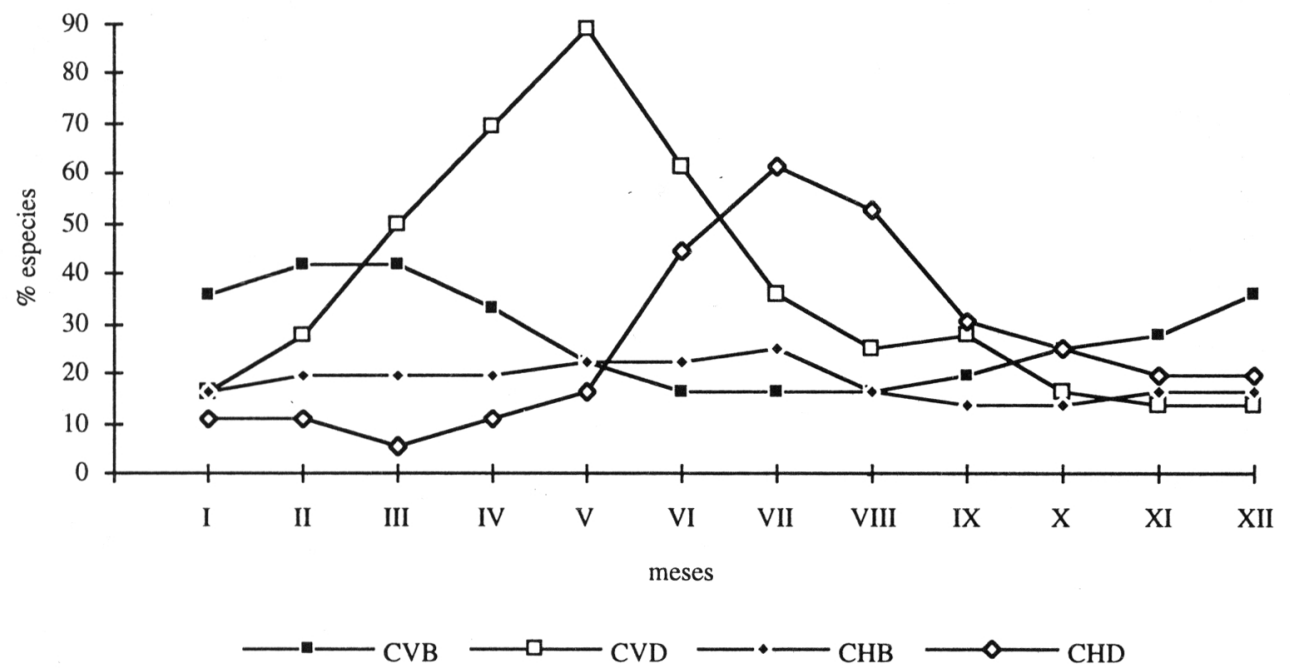

Figura 6. Porcentaje mensual de especies que presentan las fenofases vegetativas. Crecimiento vegetativo del braquiblasto (CVB), crecimiento vegetativo del dolicoblasto (CVD), caída de hojas del braquiblasto (CHB) y caída de hojas del dolicoblasto (CHD). Monthly percentage of species showing vegetative phenophases. Brachyblast vegetative growth $(C V B)$, dolichoblast vegetative growth (CVD), leaf shedding brachyblast $(\mathrm{CHB})$ and leaf shedding dolichoblast (CHD). 
destacando Junio con 11 y siendo menos significativo en Otoño (3 especies), Invierno (2 especies) y Primavera (5 especies). La estación con mayor número de especies presentando esta fenofase es el Verano (fig. 6), destacando Julio con 22, por el contrario es la Primavera la estación con menor caída de hojas, destacando Marzo con sólo 2 especies. La duración de esta fenofase varía entre 1 mes (Juniperus oxycedrus) y todo el año (Ptilostemon hispanicus) siendo de 2 a 3 meses el periodo mas común; dos especies no presentan caída apreciable de hojas (Quercus rotundifolia y Rubia peregrina) y otras tres no presentan hojas (Retama sphaerocarpa, Ulex parviflorus y Genista umbellata).

La principal época de caída de la hoja del braquiblasto (CHB) es desde Mayo a Julio (fig. 6), mientras que el mínimo ocurre en el Otoño. El período de caída oscila entre 2 meses (Erica arborea) y 12 meses (Lavandula stoechas, Phlomis purpurea, Cistus albidus, Cistus ladanifer y Teucrium fruticans), siendo el más común el de 12 meses.

La comunidad se caracteriza ecomorfológicamente por un dominio de las especies siempreverdes aunque existe un pequeño contingente caducifolio, una gran diversidad en cuanto a tipos de frutos, un predominio de las hojas malacófilas sobre las que presentan grado de esclerofilia existiendo algunas especies áfilas y el dorninio de los fanerófitos sobre el resto de tipos biológicos (tab. 1).

\section{CONCLUSIONES}

La estación mas favorable para la vegetación en la zona estudiada (tab. 2) es la Primavera, donde se concentra el máximo de especies en prefloración (PF), floración (FL) y crecimiento vegetativo del dolicoblasto (CVD); la estación más desfavorable es el Verano donde se concentra el mayor porcentaje de especies con caída de hojas del dolicoblasto (CHD) y del braquiblasto (CHB) y el menor porcentaje de especies con crecimiento vegetativo de dolicoblasto (CVD) y braquiblasto (CVB). La comunidad se com-

\begin{tabular}{ccccc}
\hline Fen. Rep. & PF & FL & FR & DS \\
\hline IF max. & Pr. (IV) & Pr. (IV) & Pr. (V) & Ot. (IX) \\
IF min. & Ot. (XI) & In. (XII-II) & Ot.-In. (X-XI)/(I-II) & In.-Pr. (XII-I)/(III-IV) \\
VF max. & Pr. (IV-V) & Pr. (V) & Ve. (VII) & Ot. (IX) \\
VF min. & Ot. (XI) & In. (II) & In. (I-II) & Pr. (III-V) \\
\hline Fen. Veg. & CVD & CHD & CVB & CHB \\
\hline IF max. & Pr. (V) & Ve. (VI) & In. (XII) & Pr.-Ve. (V) \\
IF min. & Ve.-Ot. (VII/X-XI) & In. (I-II) & Ve. (VI-VIII) & Ot.-In. (IX-X)/(XII-I) \\
VF max. & Pr. (V) & Ve. (VII) & In.-Pr. (II-III) & Ve. (VII) \\
VF min. & Ot.-In. (XI-XII) & Pr. (III) & Ve. (VI-VIII) & Ot. (IX-X) \\
\hline
\end{tabular}

Tabla 2. Caracterización anual de la comunidad (estaciones y meses) para las fenofases reproductivas (Fen. Rep.) y vegetativas (Fen. Veg.). IF: periodos con máximos (max.) o mínimos (min.) de iniciación de cada fenofase. VF: periodos con máxima (max.) o mínima (min.) concentración de especies en cada fenofase. In: Invierno; Pr: Primavera; Ve: Verano y Ot: Otoño. Community anual characterization (seasons and months) based on reproductive (Fen. Rep.) and vegetative (Fen. Veg.) phenophases. IF: maximum (max.) or minimun (min.) starting phenophase period. VF: maximum (max.) or minimun (min.) number of species showing each phenophase. In: Winter; Pr.: Spring; Ve: Summer; Ot: Autum. 
porta como multiestacional para todas las fenofases estudiadas.

La prefloración (PF), floración (FL) y crecimiento vegetativo del dolicoblasto(CVD) coinciden con un aumento significativo en las temperaturas medias mensuales, manteniéndose la precipitación en valores altos (fig. 1) e incrementándose el número de horas de sol (fig. 2). La caída de hojas del dolicoblasto (CHD) y braquiblasto (CHB) coinciden con un aumento general de la temperatura media mensual combinado con una drástica disminución de la precipitación media mensual (fig. 1). Esta caída de hojas (Verano) puede estar motivada por una adaptación al déficit hídrico que provoca la reducción parcial de hojas, a diferencia de la caída de hojas de los deciduos auténticos (caducifolios) que muestran esta fenofase en Invierno (estación más fría y con menos horas de sol).

Existe un contingente de especies que muestran un comportamiento distinto en sus fenofases respecto al comportamiento general de la comunidad, entre ellas destacan: Viburnum tinus, con inicio de prefloración (PF) otoñal y 8 meses de duración y 6 meses entre inicio de prefloración (PF) e inicio de la floración (FL); Ulex parviflorus con inicio de prefloración (PF) y floración (FL) otoñalinvernal y de 7 meses de duración; Arbutus unedo con floración (FL) otoñal e inicio de fructificación (FR) invernal y de 12 meses de duración; Juniperus oxycedrus con período de fructificación (FR) de mas de 1 año; Quercus coccifera con fructificación (FR) de 18 meses; Lavandula stoechas, Phlomis purpurea, Rosa canina y Teucrium fruticans presentan un inicio de crecimiento vegetativo del dolicoblasto (CVD) en los meses de Agosto y Septiembre y por último Bupleurum fruticosum presenta caída de hojas del dolicoblasto (CHD) en Invierno. Alguna de éstas especies (Viburnum tinus, Arbutus unedo) con comportamiento anómalo respecto a la norma de la comunidad podrían formar parte de un contingente relíctico terciario en las recientes condiciones mediterráneas (Herrera, C., 1984b, 1982; Axelrod, 1975; Raven, 1973; Domínguez Lozano et al, 1993).

Los caméfitos y nanofanerófitos xerófilos igualan en número a los meso y microfanerófitos, lo cual representa una degradación del alcornocal. La relación fanerófitos/caméfitos en la comunidad estudiada indicaría (Danin y Orshan, 1990) la incorporación a la formación boscosa de especies pertenecientes a etapas de degradación de la misma.

\section{BIBLIOGRAFIA}

AXELROD, D. L. -1975-. Evolution and biogeography of the Madrean-Tethyan sclerophyll vegetation. Ann. Missouri Bot. Gard., 62:284-334.

BRAUN-BLANQUET, J. -1979-. Fitosociología. Ed. Blume.

CABEZUDO, B., T. NAVARRO, A.V. PÉREZ LATORRE, J.M. NIETO CALDERA y G. ORSHAN -1992-. Estudios fenomorfológicos en la vegetación del sur de España. I. Cistus. Acta Botanica Malacitana, 17:229-237.

DANIN, A. \& G. ORSHAN -1990-. The distribution of Raunkiaer life forms in Israel in relation to the environment. Journal of Vegetation Science, $1: 41-48$.

DOMÍNGUEZ LOZANO, F. y F. MARTÍNEZ ATIENZA -1993-. Acerca de la distribución española de Arbutus unedo L. (Ericaceae). Bol. R. Soc. Esp. Hist. Nat. (Sec. Biol.), 89 (14): $135-161$.

EVENARI, M. E., D. SCHULZE, L. KAPPEN, U. BUSCHBOM \& O. L. LANGE - 1975-. Adaptative mechanisms in desert plants. In: Vernberg, E. J. (ed.). Physiological adaptation to the environment. pp.111-129. New York.

FLORET , C., M. J. GALAN, E. LE FLOC'H, G. ORSHAN \& F. ROMANE -1990-. Growth forms and phenomorphology traits along an enviromental gradient: tools for studying vegetation?. Journal of Vegetation Science, 1:71-80. 
HALLOY, S. -1983-. The use of convergence and divergence in the interpretation of adaptations in high mountain biota. Evolutionary theory, 6: 233-255.

HALLOY, S. -1990-. A morphological classification of plants with special reference to the New Zealand alpine flora. Journal of Vegetation Science, 1:291-304.

HERRERA, C. -1982-. Biología reproductiva de plantas leñosas mediterráneas: síndromes tropicales lejos del trópico. II Jornadas de Taxonomía Vegetal. Resúmen de conferencia. Sevilla.

HERRERA C. -1984b-. Patrones morfológicos y funcionales en plantas del matorral mediterráneo del sur de España. Studia Oecologica, 5:734.

LE ROUX, A., X. L. KYRIACOU \& G. ORSHAN -1984-. The phenomorphology of selected plants in Mediterranean-type ecosistems of South Africa. Bull. Soc. Bot. Fr. (Actual. Bot.), 131: 441-450.

MOONEY, H. A. et al -1983-. Plant form and function in relation to nutrient gradients. In: Day, J. A. (ed.) Mineral nutrients in Mediterranean ecosystems. S. African Nat. Sci. Prog. Rep., 71:55-76

NIETO CALDERA, J. M., A. V. PÉREZ LATORRE y B. CABEZUDO -1991-. Biogeografía y series de vegetación de la provincia de Málaga (España). Acta Botanica Malacitana, 16(2):417-436.

ORSHAN, G. -1982-. Monocharacter growth-form types as a tool in an analytic-synthetic study of growth-forms in mediterranean type ecosistems. A proposal for an inter-regional program. Ecol. Mediterranea, 8:159 171.

ORSHAN, G. -1986-. Plant form as describing vegetation and expresing adaptation to environment . Anali di Botanica, 54:7-37.
ORSHAN, G. (ed.) -1989a-. Plant phenomorphological studies in Mediterranean type ecosystems. Kluwer Academic Publishers. Dodrecht.

ORSHAN, G. -1989b-. Introduction In: Orshan, G. (ed.) Plant phenomorphological studies in Mediterranean type ecosystems. Kluwer Academic Publishers. Dodrecht. pp. 1-5.

PÉREZ LATORRE, A. V., J. M. NIETO CALDERA y B.CABEZUDO -1993-. Datos sobre la vegetación silicícola de Andalucía II. Los alcornocales. Acta Botanica Malacitana, 18.

PIERCE, S. M. -1984-. A synthesis of plant phenology in the fynbos biome. South African National Scientific Programmes report 88.

RAVEN, P. H. -1973-. The evolution of mediterranean floras. In: F. di Castri y $\mathrm{H}$. A. Mooney (eds.), Mediterranean-type ecosystems, pp. 213-224. Springer. New York.

Aceptado para su publicación en Mayo de 1993

Dirección de los autores. Departamento de Biología Vegetal. Facultad de Ciencias. Universidad de Málaga. Apdo. 59, 29080 Málaga. 